\title{
Cyanidin-3-O- $\beta$-glucoside regulates fatty acid metabolism via an AMP-activated protein kinase- dependent signaling pathway in human HepG2 cells
}

\author{
Honghui Guo ${ }^{1 *}$, Guoling Liu', Ruimin Zhong ${ }^{1}$, Yun Wang ${ }^{2}$, Duan Wang ${ }^{2}$ and Min Xia ${ }^{2}$
}

\begin{abstract}
Background: Hepatic metabolic derangements are key components in the development of fatty liver disease. AMP-activated protein kinase (AMPK) plays a central role in controlling hepatic lipid metabolism through modulating the downstream acetyl CoA carboxylase (ACC) and carnitine palmitoyl transferase 1 (CPT-1) pathway. In this study, cyanidin-3-O- $\beta$-glucoside (Cy-3-g), a typical anthocyanin pigment was used to examine its effects on AMPK activation and fatty acid metabolism in human HepG2 hepatocytes.

Results: Anthocyanin Cy-3-g increased cellular AMPK activity in a calmodulin kinase kinase dependent manner. Furthermore, Cy-3-g substantially induced AMPK downstream target ACC phosphorylation and inactivation, and then decreased malonyl CoA contents, leading to stimulation of CPT-1 expression and significant increase of fatty acid oxidation in HepG2 cells. These effects of Cy-3-g are largely abolished by pharmacological and genetic inhibition of AMPK.

Conclusion: This study demonstrates that Cy-3-g regulates hepatic lipid homeostasis via an AMPK-dependent signaling pathway. Targeting AMPK activation by anthocyanin may represent a promising approach for the prevention and treatment of obesity-related nonalcoholic fatty liver disease.
\end{abstract}

Keywords: anthocyanin, AMP-activated protein kinase, acetyl CoA carboxylase, carnitine palmitoyl transferase 1, fatty acid metabolism

\section{Background}

Nonalcoholic fatty liver disease (NAFLD) is a serious consequence of obesity, increasing the risk of liver cancer or cirrhosis [1]. The origin of this disease is unknown and probably multifactorial. Nevertheless, because impaired lipid metabolism is recognized as an associate and/or promoting mediator of the disease, management of hepatic metabolic disorders becomes an essential strategy for prevention and treatment of obesity-related NAFLD [2].

AMP-activated protein kinase (AMPK) is a key sensor of cellular energy status and it is also recognized as a

\footnotetext{
* Correspondence: guohh1999@hotmail.com

'Department of Food Science, Yingdong College of Bioengineering,

Shaoguan University, Shaoguan, Guangdong Province, China

Full list of author information is available at the end of the article
}

major regulator of liver and whole body lipid homeostasis [3]. AMPK activation in the liver results in the phosphorylation and inactivation of acetyl-CoA carboxylase $(\mathrm{ACC})$, a direct AMPK substrate, leading to decreased conversion of acetyl-CoA to malonyl CoA [4]. AMPK activation also results in phosphorylation and activation of malonyl CoA decarboxylase (MCD), resulting in further lowering of malonyl CoA levels. Malonyl CoA allosterically inhibits carnitine palmitoyl-CoA transferase 1 (CPT-1), the enzyme responsible for transport of long chain acyl-CoAs into mitochondria for oxidation. Additionally, as malonyl CoA is required for de novo synthesis of fatty acids, decreased malonyl CoA leads to a reduction in hepatic fatty acid synthesis [5]. Therefore, AMPK activation leads to a concomitant inhibition of fatty acid synthesis and activation of fatty acid oxidation.

\section{C) Biomed Central}


Anthocyanins are naturally occurring polyphenolic compounds in the plant foods and widely distributed in fruits, vegetables, and pigmented cereals, suggesting that we can ingest significant amounts of anthocyanins from plant-based daily diets [6]. Clinical investigations have indicated that the moderate consumption of anthocyanins through the intake of lyophilized grape powder or berry-derived anthocyanin supplements is associated with improved lipid profile $[7,8]$. In previous animal studies from us and other investigators, anthocyanins have been shown to ameliorate dyslipidemia and hepatic steatosis in different rodent models [9-12]. However, the molecular mechanism under this action remains unknown and needs further investigation in cells.

Considering the key role of AMPK activation in regulating lipid metabolism and the potential capability of anthocyanin in preventing hepatic steatosis, we observed the influence of cyanidin-3-O- $\beta$-glucoside (Cy-3-g), the most abundant anthocyanin in plants [6], on AMPK activation and fatty acid metabolism in human HepG2 hepatocytes. The signal pathway of the action involved in AMPK molecular activation was also investigated.

\section{Results}

\section{Anthocyanin induces AMPK activation in HepG2 cells}

To assess the impact of anthocyanin on AMPK activation, the human HepG2 cells were treated with Cy-3-g of $1 \sim 100 \mu \mathrm{M}$ or with an AMPK activator-AICAR (1 $\mathrm{mM}$ ) for $1 \mathrm{~h}$, respectively. We first determined the Thr172 phosphorylation of AMPK because this site is an essential marker of AMPK activity. Cy-3-g significantly stimulated AMPK phosphorylation in a concentrationdependent fashion over the untreated control (Figure 1A). Importantly, the effect of $\mathrm{Cy}-3-\mathrm{g}$ at a concentration of $10 \mu \mathrm{M}$ was comparable with AICAR. However, no change in the expression of endogenous total AMPK protein was noted. To further confirm that the effect of anthocyanin on AMPK, the kinase activity of AMPK was assayed by using SAMS peptide as the substrate. Consistent with the increased AMPK phosphorylation, Cy-3-g strongly stimulates AMPK kinase activity in a concentration-dependent manner in HepG2 cells (Figure 1B).

The enzymes fructose1,6-diphosphatase (FBPase) and glycogen phosphorylase (GPPase) are allosterically modulated by AMP. AMP activates GPPase, whereas it inhibits FBPase [13]. To assess the specificity of anthocyanin for AMPK activation, we determined their effects on these two enzymes. Anthocyanin treatment had no effect on GPPase activity at concentrations up to $1 \mathrm{mM}$, while the $\mathrm{EC}_{50}$ of AMP for the activation of GPPase was $18.5 \mu \mathrm{M}$ (Figure 1C). Similarly, anthocyanin showed no significant inhibitory effect on FBPase at concentrations up to $1 \mathrm{mM}$, while AMP significantly inhibited FBPase
$\left(\mathrm{IC}_{50}=1.6 \mu \mathrm{M}\right.$, Figure $\left.1 \mathrm{D}\right)$. These results strongly indicate that anthocyanin stimulates AMPK activity in a manner that differs from AMP. To further address this issue, we evaluated the additivity of AMP and anthocyanin mediated AMPK stimulation. Anthocyanin Cy-3-g increased AMPK activity in the presence of a saturating concentration of AMP (Figure 1E) and AMP stimulated AMPK in the presence of a maximally efficacious concentration of Cy-3-g (Figure 1F). This further supports the hypothesis that anthocyanin binds at a unique site that differs from that of AMP binding.

\section{Intracellular $\mathrm{Ca}^{2+}$ and CaMKK- $\beta$ mediates the anthocyanin-induced activation of AMPK}

AMPK is also activated by calmodulin-dependent protein kinase kinase $\beta$ (CaMKK- $\beta$ ) triggered by a rise in intracellular calcium ions $\left(\left[\mathrm{Ca}^{2+}\right]_{\mathrm{i}}\right)$, without detectable changes in the AMP/ATP ratio [14]. We next investigated whether the $\mathrm{Ca}^{2+}$ signaling was involved in the AMPK activation upon exposure of HepG2 cells to anthocyanin. Anthocyanin exposure resulted in a rapid and transient increase in $\left[\mathrm{Ca}^{2+}\right]_{\mathrm{i}}$ concentration (Figure 2A). Chelation of intracellular $\mathrm{Ca}^{2+}$ with BAPTA-AM prevented the anthocyanin-induced phosphorylation of AMPK (Figure 2B), suggesting that intracellular $\mathrm{Ca}^{2+}$ was involved in the signaling cascade initiated by the anthocyanin. Furthermore, preincubation of HepG2 cells with STO-609, a CaMKK- $\beta$ inhibitor, decreased baseline of AMPK activity, and anthocyanin did not increase AMPK activation (Figure $2 \mathrm{C}$ ), suggesting a role for CaMKK- $\beta$. To confirm that CaMKK- $\beta$ was the kinase upstream of AMPK, a siRNA approach was utilized to knock down CaMKK- $\beta$ expression in HepG2 cells. Similar to the effects of STO-609, baseline AMPK activity was reduced and remained unchanged upon exposure to anthocyanin (Figure 2D).

\section{Anthocyanin suppresses ACC activity, decreases malonyl CoA contents and stimulates CPT-1 expression in HepG2 cells}

To determine whether AMPK activation affects its downstream target proteins, we examined the activity of ACC, a key enzyme in the regulation of fatty acid metabolism [5]. Anthocyanin suppressed ACC activity in a dose-dependent fashion in HepG2 cells (Figure 3A). These effects are probably caused by phosphorylation of ACC by AMPK, as ACC serine-79 phosphorylation was increased after anthocyanin treatment, whereas overall ACC protein expression did not change (Figure $3 \mathrm{~B}$ ). AMPK activation and ACC inhibition may lead to a reduction in malonyl CoA levels and release of CPT-1 from allosteric inhibition $[15,16]$. We next investigated the net effect of these changes in malonyl CoA. Our results show that malonyl CoA levels decreased acutely 
A

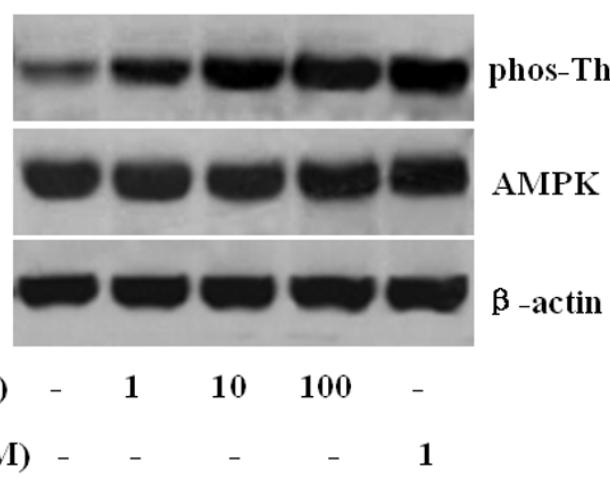

$\operatorname{AICAR}(\mathrm{mM}) \quad-\quad-\quad-\quad-\quad-1$
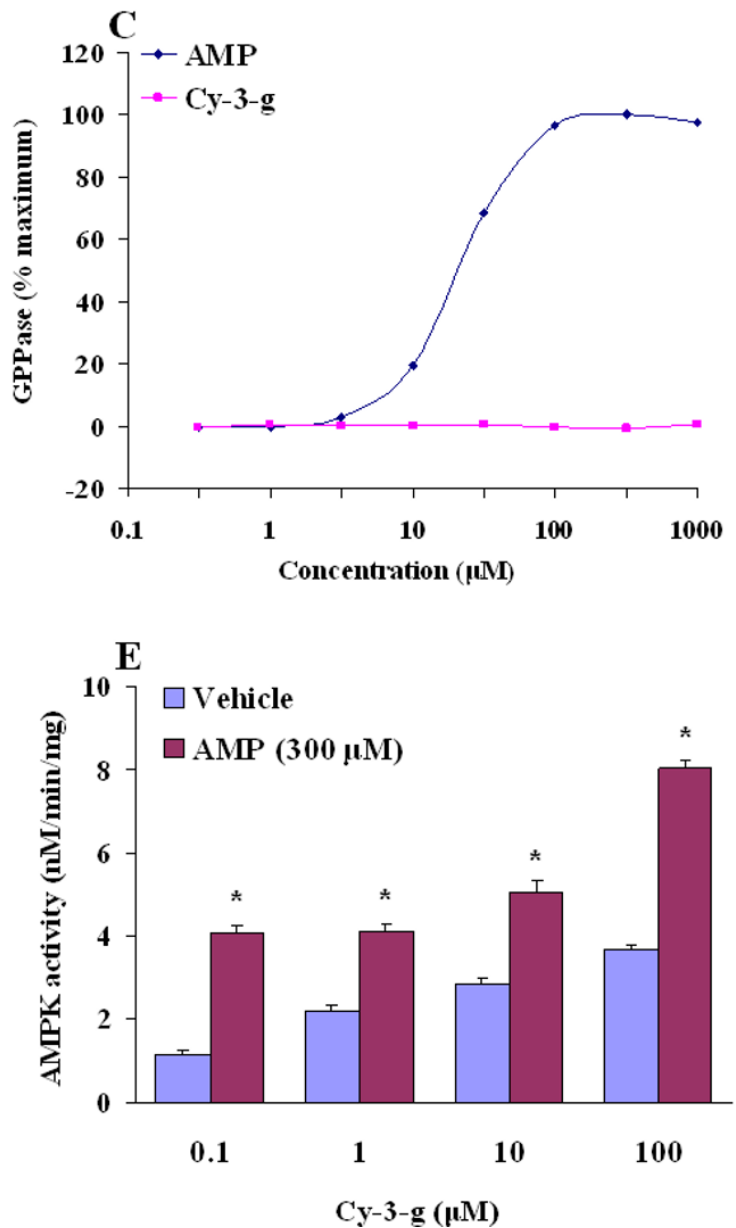

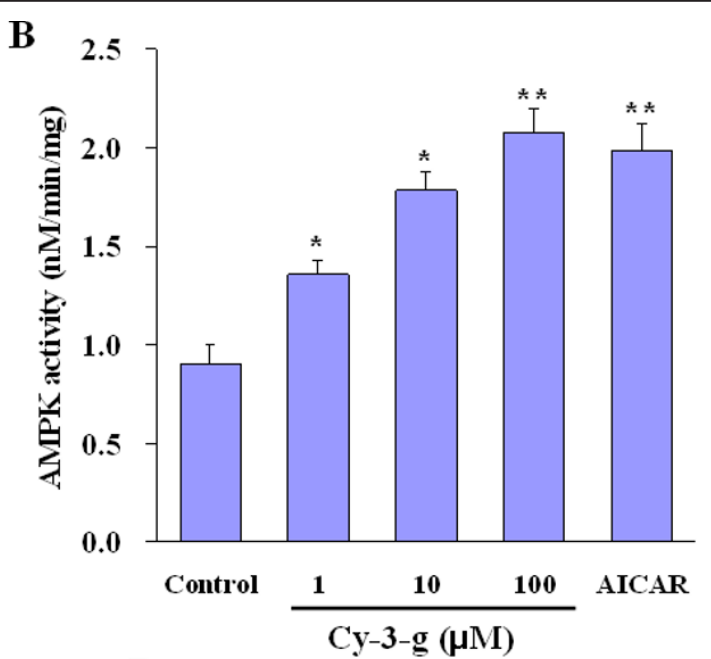

D
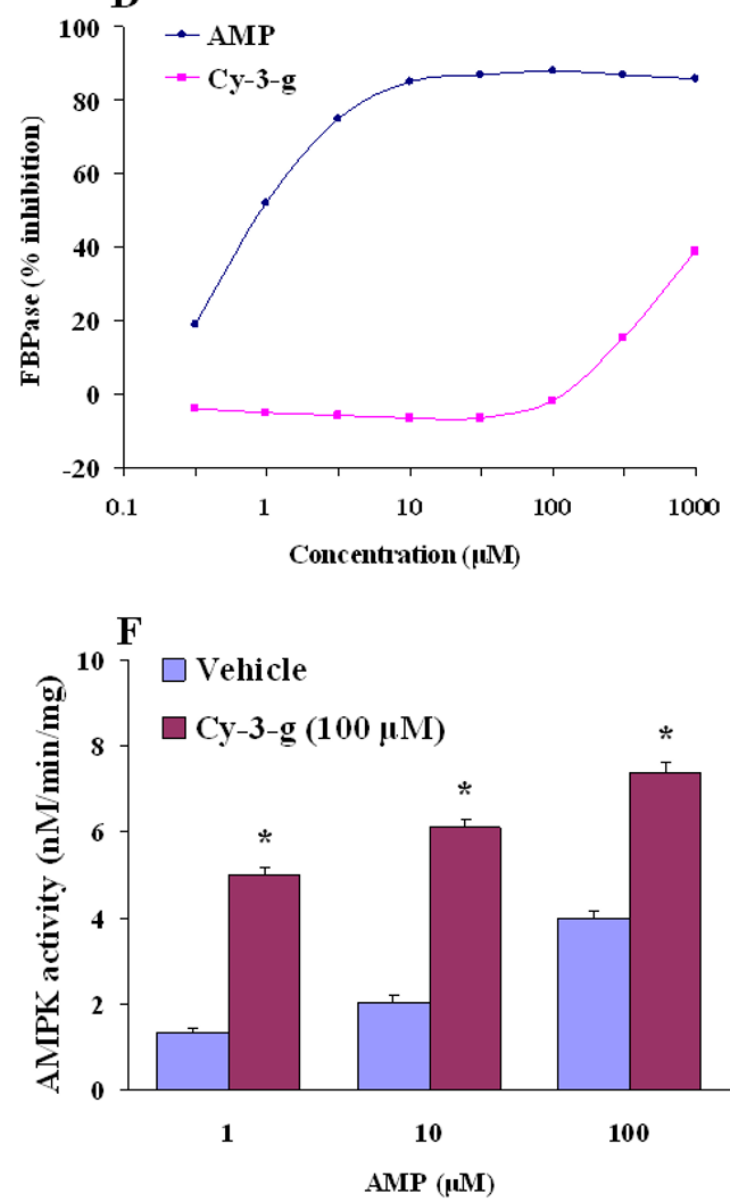

Figure 1 Anthocyanin activates AMPK in cultured HepG2 cells. (A) HepG2 cells were treated with various concentrations of Cy-3-g or AICAR ( $1 \mathrm{mM}$ ) for $1 \mathrm{~h}$. The cells were then lysed, and $100 \mu \mathrm{g}$ of the cell lysates underwent SDS-PAGE followed by Western blot analysis for AMPK phosphorylation. (B) Two microgram of protein extracts were subjected to AMPK activity assays with SAMS peptide as substrates, results are expressed as nmoles ATP/mg protein/min. ${ }^{*} P<0.05$ or ${ }^{* *} P<0.01$ compared to control. (C) GPPase activity was measured in the presence of glycogen using a phosphoglucomutase and glucose-6 phosphate dehydrogenase-coupled spectrophotometric method. Effects of anthocyanin are expressed as a percent of the maximum stimulation achieved by AMP. (D) FBPase activity was determined using the substrate D-fructose-1,6bisphosphate and colorimetric detection of free phosphate. Results are expressed as percent inhibition relative to vehicle control. (E) The concentration-dependent AMPK activation by anthocyanin and additive effects in the presence of $300 \mu \mathrm{M}$ AMP. ${ }^{*} P<0.05$ compared to vehicle. (F) AMP dose-responsive AMPK activation and additive effects in the presence of $100 \mu \mathrm{M} C y-3-g .{ }^{*} P<0.05$ compared to vehicle. The results presented in panels (B) through (F) are means \pm SE from experiments run in triplicate and are representative of at least three independent experiments. 


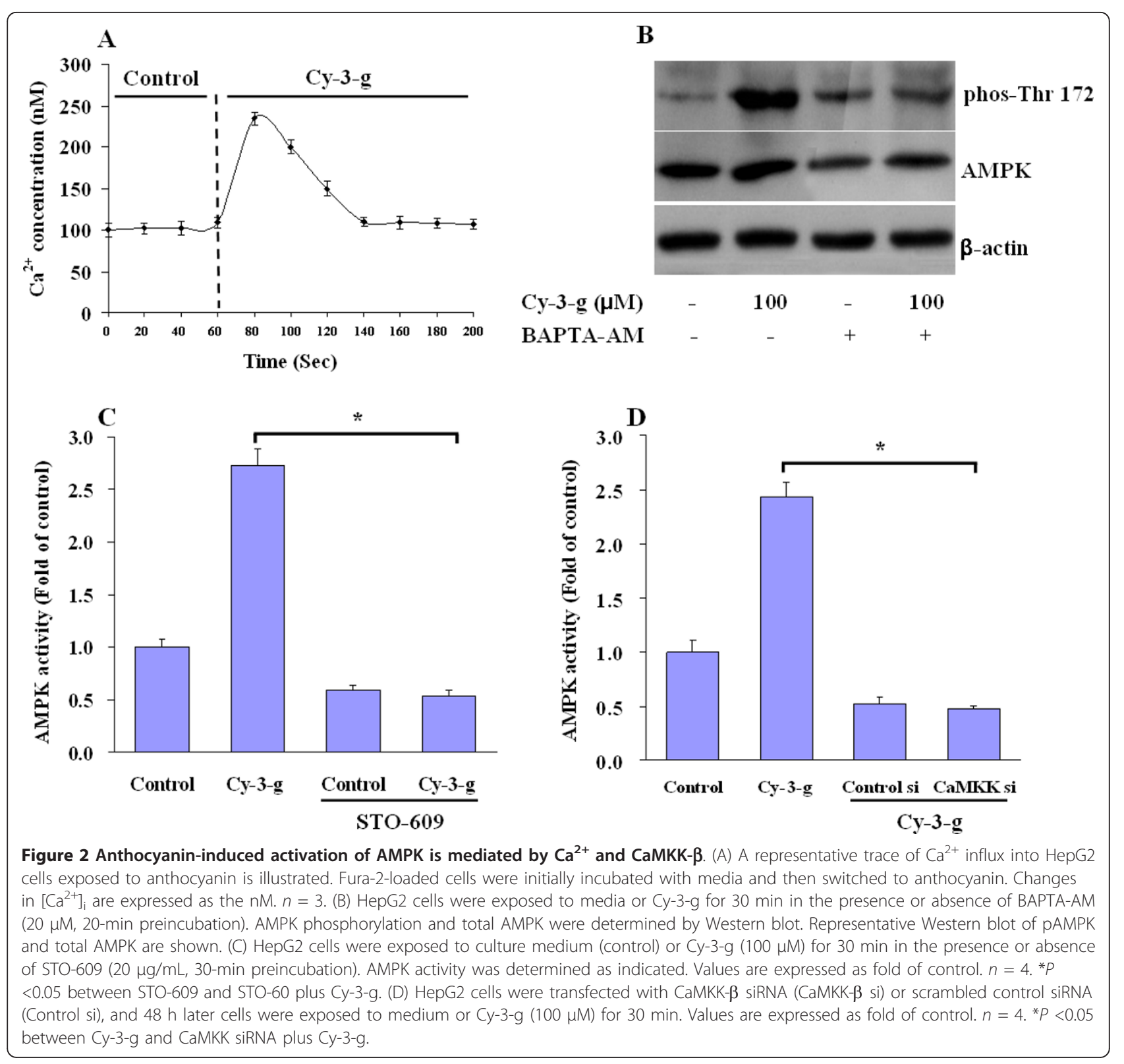

after anthocyanin treatment in a dose-dependent fashion (Figure 3C), accompanying with a statistically significant and dose-responsive increase of CPT-1 mRNA expression in HepG2 cells (Figure 3D).

\section{Anthocyanin increases fatty acid oxidation and inhibits lipogenesis in HepG2 cells}

Since AMPK activation inhibits ACC activity, further leading to reduction of malonyl $\mathrm{CoA}$ and promotion of CPT-1 activation, one expected consequence is the increased fatty acid oxidation and reduced lipogenesis. To evaluate this hypothesis we studied the effects of anthocyanin on hepatic fatty acid oxidation and synthesis. Anthocyanin functionally enhanced fatty acid oxidation as $\mathrm{Cy}-3-\mathrm{g}$ increased palmitate oxidation rates in HepG2 cells, with $\mathrm{EC}_{50}=5.7 \mu \mathrm{M}$ for Cy-3-g (Figure 4A). In addition, anthocyanin treatment significantly inhibited the fatty acid synthesis as measured by incorporation of $\left[{ }^{14} \mathrm{C}\right]$ acetate into fatty acids, with $\mathrm{IC}_{50}=6.3$ $\mu \mathrm{M}$ for Cy-3-g (Figure 4B). The effect of anthocyanin on lipogenesis may be attributed to reduced fatty acid synthase (FAS) gene expression, as Cy-3-g treatment caused a dose-dependent inhibition of FAS mRNA expression in HepG2 cells (Figure 4C). Further measurement showed that Cy-3-g treatment caused a dosedependent reduction of triglyceride (TG) content in HepG2 cells (Figure 4D). Inhibition of mitochondrial fatty acid oxidation with etomoxir was enough to 


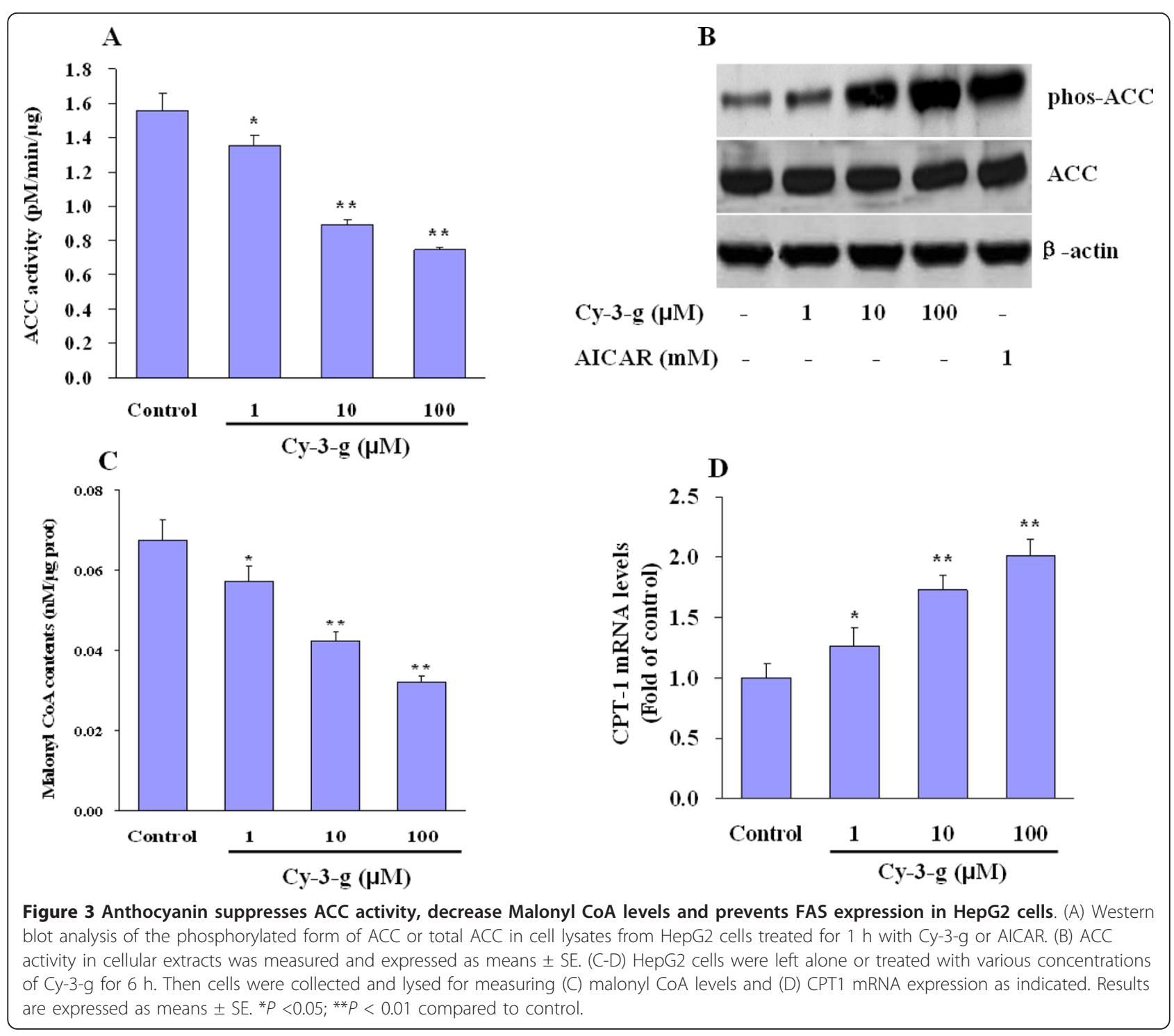

hamper the decrease in triglyceride levels induced by anthocyanin (Figure 4E), indicating that AMPKmediated mitochondrial $\beta$-oxidation attributed for the major effects of anthocyanin. To exclude the possibility that inhibition of fatty acid metabolism could be mediated in part by cytotoxicity, we assessed the potential cellular cytotoxicity of anthocyanin. Treatment of human HepG2 cells with Cy-3-g at the concentrations up to $100 \mu \mathrm{M}$ showed no measurable cytotoxicity, whereas staurosporine, a known cytotoxic agent, significantly increased cellular cytotoxicity (Figure 4F). We further probed the mechanism of action by assessing whether anthocyanin alters the cellular levels of AMP and ATP. Cy-3-g treatment at concentrations up to 100 $\mu \mathrm{M}$ caused no significant alterations in ATP and AMP levels in HepG2 cells (Table 1). Taken together, these results indicate the anthocyanin exert their cellular effects by direct activation of AMPK activity and not as a consequence of cellular toxicity, or by altering the AMP/ATP ratio.

\section{AMPK signaling mediates anthocyanin-induced reduction of lipid levels in HepG2 cells}

To elucidate the AMPK pathway involved in regulating hepatic fatty acid metabolism, we applied several independent approaches to inhibit AMPK activation. Pretreatment of HepG2 cells with compound C, a potent and nonspecific inhibitor of AMPK [17], significantly prevented anthocyanin-mediated inhibition of ACC activity (Figure 5A) and FAS expression (Figure 5B) in HepG2 cells. To examine the critical role of AMPK in anthocyanin-mediated hepatic fatty oxidation, we used the specific siRNA against AMPK. Expression of endogenous AMPK protein was remarkably suppressed by 

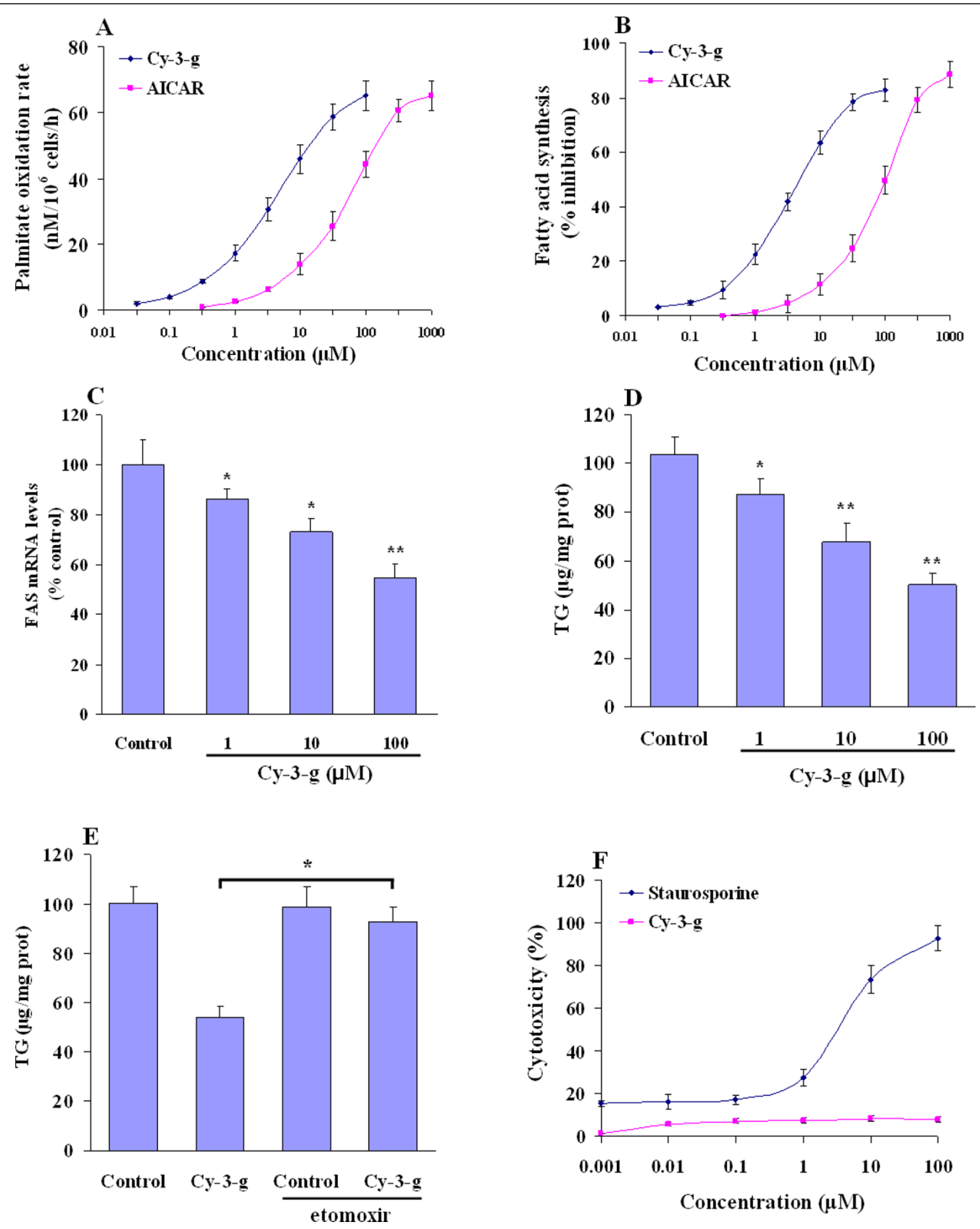

Figure 4 Anthocyanin increases fatty acid oxidation in HepG2 cells. (A) Fatty acid oxidation rate was determined by cotreatment with various concentrations of $\mathrm{Cy}-3-\mathrm{g}$ in the presence of $\left[{ }^{14} \mathrm{C}\right]$ palmitate. Results are expressed as fold of control from at least three independent experiments. (B) Fatty acid synthesis was measured as incorporation of ${ }^{14} \mathrm{C}$-acetate into fatty acids in HepG2 cells during a 6-h treatment with increasing concentrations of Cy-3-g or AICAR, respectively. Results are given as percent inhibition of fatty acid synthesis relative to the control and are means \pm SE from triplicate analysis. (C) Dose-dependent inhibition of FAS mRNA expression by Cy-3-g. The results are expressed as percent of control from experiments run in triplicate of three independent experiments. (D) Intracellular TG contents were measured in cell lysates by spectrophotometic methods. Results are means \pm SE from triplicate measurements. ${ }^{*} P<0.05$; ${ }^{* *} P<0.01$ compared with control. (E) HepG2 cells pre-incubated with vehicle or etomoxir $(50 \mathrm{mM})$ for $1 \mathrm{~h}$ were treated with either vehicle (control) or Cy-3-g (100 $\mu \mathrm{M})$. Then, Intracellular triglyceride contents were determined. ${ }^{*} P<0.05$ between Cy-3-g and Cy-3-g plus etomoxir. (F) The cellular cytotoxity assays run in HepG2 cells using MTS reagents comparing a known cytotoxin-staurosporine and Cy-3-g. Data are from a minimum of 4 experiments run in triplicate and is demonstrated as means $\pm \mathrm{SE}$. 
Table 1 Nucleotide levels following treatment of HepG2 with Cy-3-g

\begin{tabular}{lccc}
\hline $\begin{array}{l}\text { Cy-3-g } \\
\text { concentration }\end{array}$ & $\begin{array}{c}\text { AMP } \\
\text { (nM/10 } \\
\text { cells) }\end{array}$ & $\begin{array}{c}\text { ATP } \\
\text { (nM/10 } \\
\text { cells) }\end{array}$ & $\begin{array}{c}\text { AMP/ATP } \\
\text { ratio }\end{array}$ \\
\hline Untreated control & $0.348 \pm 0.021$ & $6.236 \pm 0.160$ & $0.056 \pm 0.003$ \\
$1 \mu \mathrm{M}$ & $0.336 \pm 0.044$ & $6.525 \pm 0.129$ & $0.051 \pm 0.007$ \\
$10 \mu \mathrm{M}$ & $0.398 \pm 0.018$ & $6.271 \pm 0.087$ & $0.064 \pm 0.002$ \\
$100 \mu \mathrm{M}$ & $0.354 \pm 0.011$ & $6.633 \pm 0.310$ & $0.054 \pm 0.003$ \\
$1 \mathrm{mM}$ & $0.347 \pm 0.021$ & $6.412 \pm 0.235$ & $0.054 \pm 0.006$ \\
\hline
\end{tabular}

AMPK siRNA (Figure 6A), suggesting that the knockdown effect is specific. Compared to control siRNA, AMPK siRNA transfection reversed the phosphorylation of ACC induced by $\mathrm{Cy}-3-\mathrm{g}$ (Figure 6B), indicating that ACC activity is mainly dependent on the presence of AMPK. In line with this, direct measurement of palmitate oxidation (Figure 6C) confirmed that the effects of anthocyanin on CPT-1 expression (Figure 6D) and malonyl CoA levels (Figure 6E) were blunted in HepG2 cells where AMPK expression was knocked down. The lack of AMPK hence alters the long-term actions of anthocyanin on lipid metabolism (Figure 6F).

To further rule out that AMPK could participate to sustain the action of anthocyanin, adenoviral overexpression of dominant-negative AMPK mutants (Ad-DNAMPK) was infected into HepG2 cells. The lack of AMPK significantly compromised the anthocyaninmediated reduction of malonyl CoA levels (Figure 7A). As a consequence, the DN-AMPK completely blunted the effect of anthocyanin to increase CPT-1 mRNA expression (Figure 7B) and fatty acid oxidation (Figure $7 C)$, reversing the inhibition of triglyceride accumulation by anthocyanin (Figure 7D). Together, these data indicate that, to a large extent, AMPK functions as a novel downstream effector in regulation of lipid metabolism by anthocyanin.

\section{Discussion}

This study demonstrates that $\mathrm{Cy}-3-\mathrm{g}$, a typical anthocyanin pigment, stimulates AMPK activation via CaMKK in HepG2 cells. The increase in AMPK activation by anthocyanin suppresses ACC activity, causes the reduction in malonyl CoA levels and stimulation of CPT-1, leading to enhanced fatty acid $\beta$-oxidation and finally inhibiting the lipid accumulation in HepG2 cells. Thus, the novel findings from the present study is that anthocyanin beneficially regulates hepatic fatty acid metabolism through AMPK-ACC-malonyl CoA-CPT1 pathway.

Previous studies had shown that Cy-3-g activated AMPK and enhanced adipocytokine secretion and adipocyte-specific gene expression in isolated adipocytes $[18,19]$. Recently, Takikawa et al. have demonstrated that anthocyanin-rich bilberry extract effectively ameliorates hyperglycemia and insulin sensitivity via activation of AMPK in diabetic mice [20]. The results of these studies suggest that anthocyanin may be a potential AMPK activator. However, the impact of anthocyanin on hepatic AMPK and its-mediated physiological consequences on fatty acid metabolism is still undefined. In this study, we identified that anthocyanin Cy-3-g is a potent activator of AMPK in hepatocytes. Meanwhile, GPPase and FBPase activity were not altered in response to anthocyanin, implying that anthocyanin activated AMPK via a unique pathway unlike AICAR/AMP.

In mammals, LKB1 and CaMKK- $\beta$ have been identified as upstream kinases of AMPK, since both kinases can phosphorylate Thr172 and thus activate AMPK [14,21]. The phosphorylation of Thr172 by LKB1 requires an increase in AMP, while phosphorylation of
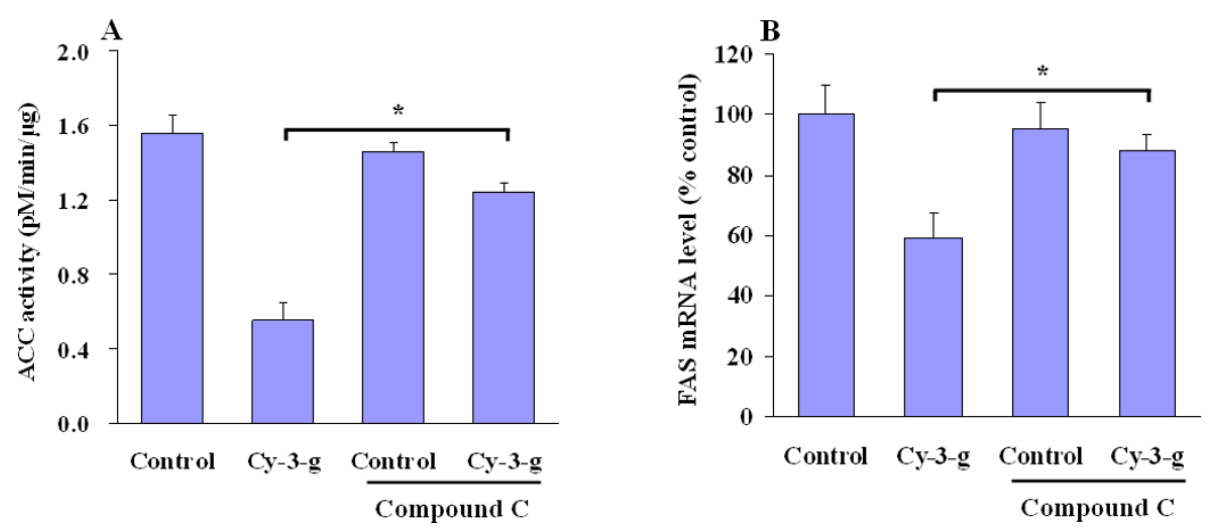

Figure 5 AMPK inhibition abrogates anthocyanin-mediated increase of ACC phosphorylation and decrease of FAS expression. HepG2 cells were treated with Cy-3-g $(100 \mu \mathrm{M})$ in the absence or presence of AMPK inhibitor compound C (10 mM). (A) ACC activity and (B) FAS mRNA expression were analyzed as indicated. Results are expressed as means \pm SE. ${ }^{*} P<0.05$ between Cy-3-g and Cy-3-g plus compound C. 


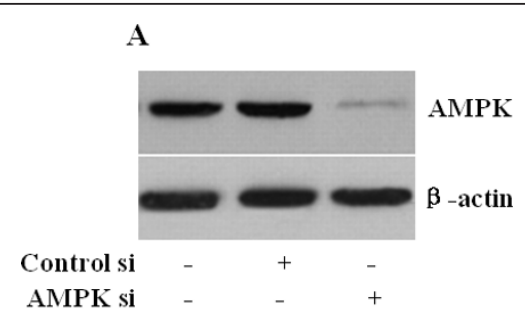

B
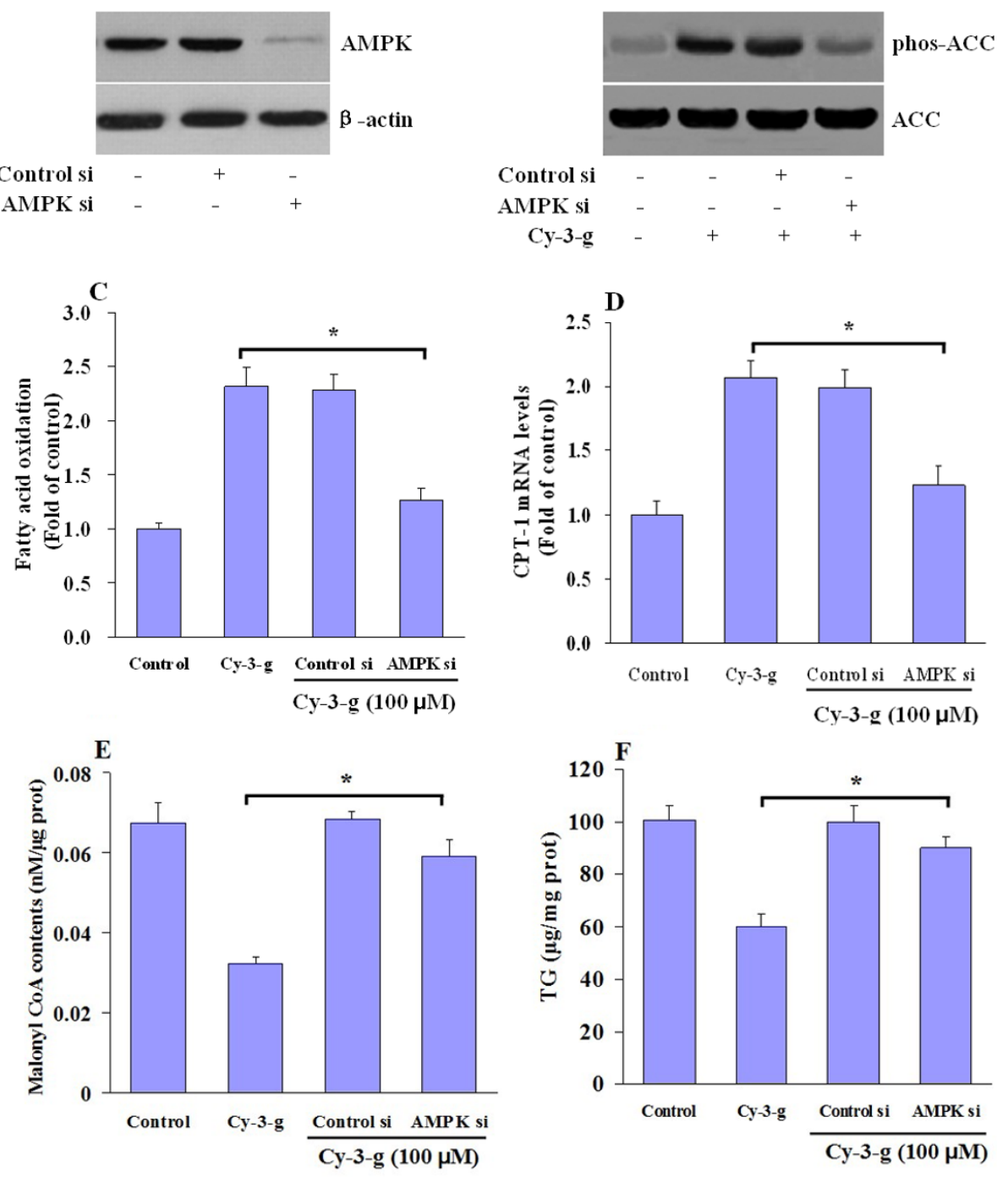

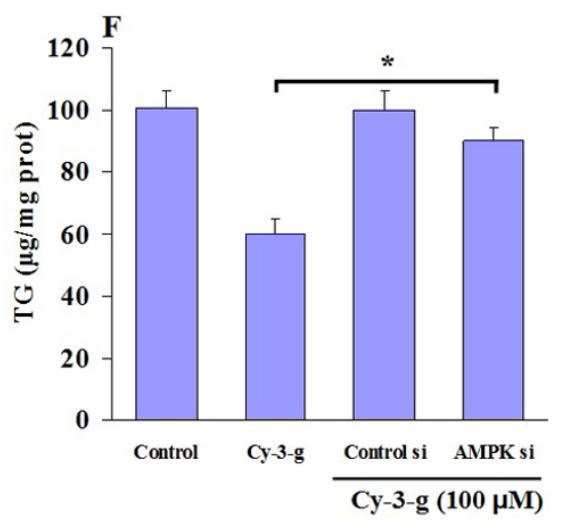

Figure 6 AMPK mediates anthocyanin-induced lipid reduction in HepG2 cells. (A) HepG2 cell were transfected with AMPK siRNA. Control siRNA was used as control. AMPK protein expression was determined by Western blot analysis. Representative blots from three independent assays were shown. (B-E) HepG2 cells were transfected with either control siRNA (Control si) or AMPK specific siRNA (AMPK si), followed by

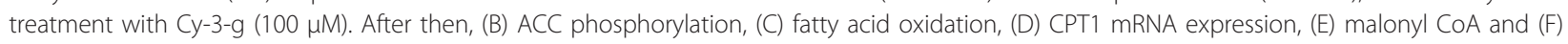
hepatic triglyceride were measured and results are shown as means \pm SE from triplicate measurements from at least three independent experiments. ${ }^{*} P<0.05$ between $C y-3-g$ and Cy-3-g plus AMPK siRNA.

Thr172 by CaMKK- $\beta$ is independent of AMP levels and is triggered by a rise in intracellular $\mathrm{Ca}^{2+}$. We found that exposing HepG2 cells to anthocyanin initiated a rapid and transient influx of $\mathrm{Ca}^{2+}$ into the cells and that AMPK activation by anthocyanin was prevented by the intracellular $\mathrm{Ca}^{2+}$-chelating agent, BAPTA-AM, suggesting that the anthocyanin-induced signaling pathway was dependent upon intracellular $\mathrm{Ca}^{2+}$. Since CaMKK- $\beta$ mediated stimulation of AMPK required an increase in intracellular $\mathrm{Ca}^{2+}$ levels, we hypothesized that CaMKK- $\beta$ may be the upstream kinase induced by anthocyanin. Indeed, the CaMKK inhibitor STO-609 decreased the baseline AMPK activity and prevented activation of the AMPK upon anthocyanin exposure. Moreover, when transfected with CaMKK- $\beta$ siRNA, both baseline and anthocyanin-induced activity of AMPK were inhibited. These data suggest that the anthocyanin-induced activation of AMPK was mediated by CaMKK- $\beta$ independently of LKB1 signaling pathway.

In the liver, AMPK coordinates the changes in the activity of enzymes of lipid metabolism and regulates the partitioning of fatty acid both in oxidative and biosynthetic pathways [22]. In accordance with this, we observed that anthocyanin treatment induced ACC 


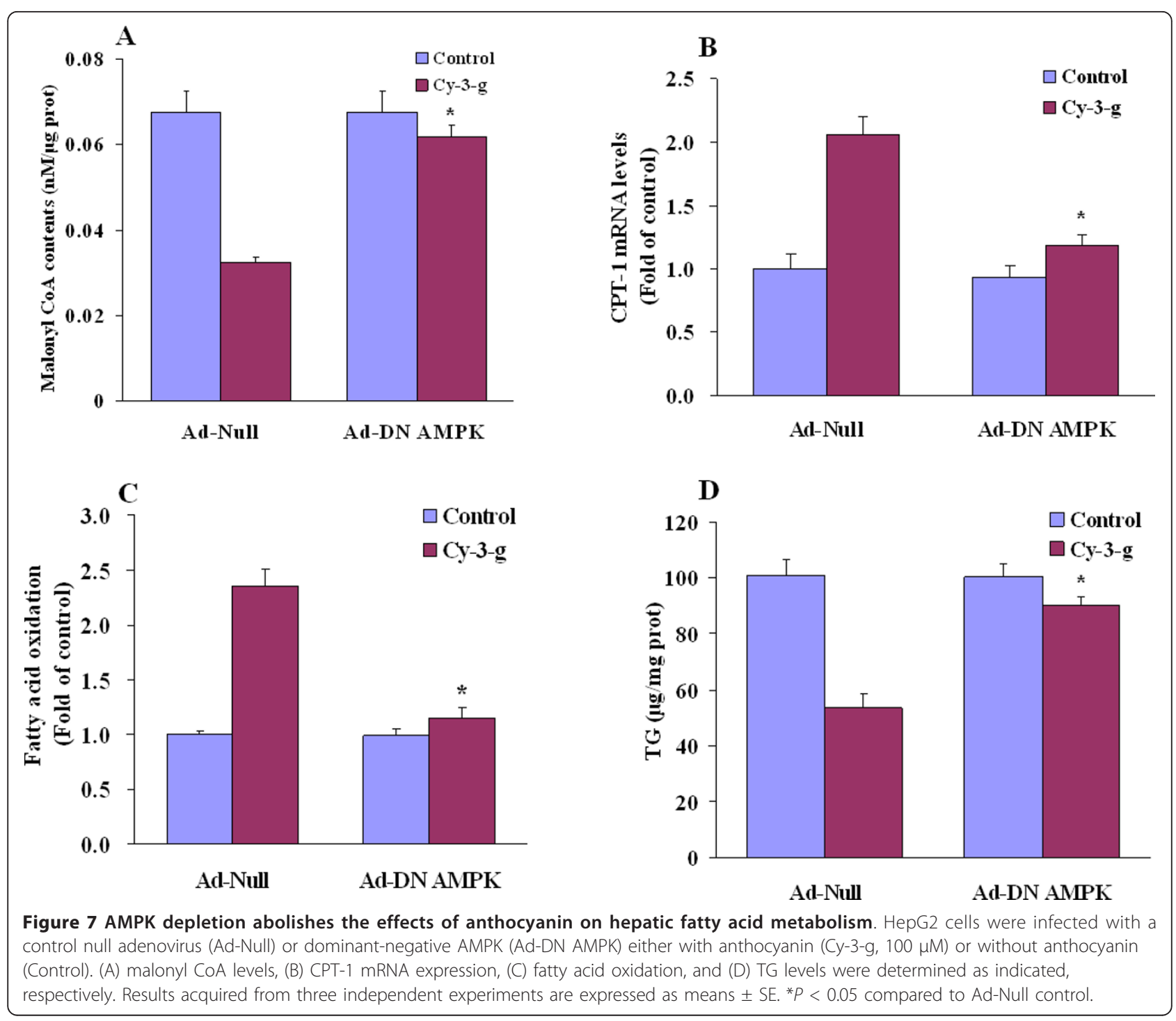

phosphorylation, depressed its enzymetic activity in HepG2 cells. Inhibition of ACC by AMPK leads to a fall in malonyl CoA content and a subsequent decrease in fatty acid synthesis and increase in mitochondrial fatty acid oxidation via the allosteric regulation of CPT-1, which catalyses the entry of long-chain fatty acyl-CoA into mitochondria [14]. Our results show that malonyl CoA is robustly decreased after anthocyanin treatment, as a result of AMPK and ACC phosphorylation. This resulted in a decrease in malonyl CoA levels associated with increased CPT-1 expression, which resulted in increased fatty acid oxidation. The ability of anthocyanin to stimulate AMPK and reduce hepatocellular lipid accumulation was abolished by pharmacological inhibition or knockdown of AMPK. Thus, anthocyanin-dependent AMPK activation functionally inhibits ACC and FAS which are two key downstream regulators of
AMPK in the control of lipid metabolism and accumulation, possibly through increased fatty acid oxidation.

\section{Conclusion}

In summary, our present study provide the mechanistic data that activation of hepatic AMPK by anthocyanin acts upstream on lipid metabolism by activating the pathways controlling the oxidation of fatty acids. Although it appears hard to get the concentration of anthocyanin shown in this study from daily consumed foods, our findings provide a novel insight into the therapeutic implications of anthocyanin in obesity-related liver disorders.

\section{Methods}

\section{Reagents}

Anthocyanin cyanidin-3-O- $\beta$-glucoside (Cy-3-g; HPLC grade) was provided by Polyphenol AS (Sandnes, 
Norway). Dulbecco's modified Eagle's medium (DMEM), fetal bovine serum (FBS), and antibiotic mixture (penicillins-treptomycin) were purchased from the Gibco BRL (Grand Island, NY). 5-aminoimidazole-4-carboxamide 1-ribofuranoside (AICAR), anti-phospho-AMPK Thr-172, anti-phospho-ACC Ser-79 and anti- $\beta$-actin were purchased from Cell Signaling Technology, Inc. (Danvers, MA). AMPK inhibitor compound $\mathrm{C}$ and calmodulin-dependent kinase kinase (CaMKK) inhibitor STO-609 were obtained from Calbiochem (San Diego, CA). The specific AMPK-targeted SAMS peptide used in AMPK activity assay was purchased from GenScript (Piscataway, NJ); siRNA against CaMKK- $\beta$ was from Qiagen, Inc. (Valencia, CA); and all other chemicals, unless otherwise specified, from Sigma-Aldrich (St. Louis, MO).

\section{Cell culture}

HepG2 cells (human hepatoma cell line, ATCC \#HB 8065, American Type Culture Collection) were cultured in DMEM supplemented with $10 \%$ FBS and antibiotics at $37^{\circ} \mathrm{C}$ in a humidified, $5 \% \mathrm{CO}_{2} / 95 \%$ air atmosphere. After reaching $\sim 80 \%$ confluence, cells were serumstarved for $24 \mathrm{~h}$ in medium containing $0.5 \%$ FBS.

\section{Adenoviruses}

The adenoviral vector expressing a dominant-negative AMPK $\alpha$ mutant (Ad-DN-AMPK) was kindly donated by Dr J. Ha (Department of Molecular Biology, Kyung Hee University, College of Medicine, Seoul, Korea).

\section{AMPK activity}

AMPK activity was measured by monitoring phosphorylation of the SAMS peptide substrate as previously described [13]. To determine whether compoundinduced AMPK activation occurs in a reversible manner, AMP or Cy-3-g were preincubated with rat liver AMPK for $10 \mathrm{~min}$ at 20 times standard assay concentrations prior to dilution and measurement of AMPK activity.

\section{Glycogen phosphorylase assay}

The rabbit glycogen phosphorylase (GPPase) at the concentration of $1.5 \mu \mathrm{g} / \mathrm{mL}$ of was added to a reaction mixture containing $20 \mathrm{mM} \mathrm{Na} \mathrm{HPO}_{4}(\mathrm{pH} 7.2), 2 \mathrm{mM}$ $\mathrm{MgSO}_{4}, 1 \mathrm{mM} \beta$-NADP $(\beta$-nicotinamide adenine dinucleotide phosphate), 1.4 U/mL G-6-PDH (glucose-6phosphate-dehydrogenase) and $3 \mathrm{U} / \mathrm{mL}$ PGM (phosphoglucomutase). AMP or Cy-3-g was added to the assay medium at the specified concentrations followed by the addition of glycogen (final concentration $1 \mathrm{mg} / \mathrm{mL}$ ) to initiate the reaction. After incubating $10 \mathrm{~min}$ at $25^{\circ} \mathrm{C}$, GPPase activity was assessed by measuring absorbance at $340 \mathrm{~nm}$ [23].

\section{Fructose 1,6-bis-phosphatase assay}

D-fructose-1,6 diphosphatase (FBPase, $0.01 \mathrm{U} / \mathrm{mL}$ final concentration) in $2 \times$ assay buffer, $100 \mathrm{mM}$ Tris $(\mathrm{pH}$ 7.0), $4 \mathrm{mM} \mathrm{MgCl}_{2}, 300 \mathrm{mM} \mathrm{NaCl}, 0.2 \mathrm{mg} / \mathrm{mL} \mathrm{BSA}$ and $6 \mathrm{mM}$ DTT, was added to test compounds to give final concentrations as indicated. Substrate, D-fructose-1,6diphosphate, at a final concentration of $0.1 \mathrm{mM}$ was added to the reaction and the reaction mix was incubated at $30^{\circ} \mathrm{C}$ for $20 \mathrm{~min}$. Following the incubation 2 volumes of Malachite Green solution (Upstate Inc., Waltham, MA) containing $0.001 \%$ Tween 20 was added and absorbance at $640 \mathrm{~nm}$ read immediately [24].

\section{Measurement of intracellular ATP and AMP levels}

To measure adenine nucleotide concentrations in HepG2 cells, each well of HepG2 cells in 6-well plates was harvested in $300 \mu \mathrm{L}$ of lysis buffer containing $5 \%$ (v/v) perchloric acid. After sonication for $5 \mathrm{~s}$, the samples were centrifuged at $16,000 \times \mathrm{g}$ for $10 \mathrm{~min}$ at $4^{\circ} \mathrm{C}$ twice to remove acid-insoluble material. The supernatant neutralized with $\mathrm{KOH}$ and analyzed by HPLC (Waters, Milford, MA) with an LC-18T reverse-phase column (Supelco, Bellefonte, PA) at a flow rate of $1 \mathrm{~mL} /$ min. Nucleotides were detected by their absorbance at $254 \mathrm{~nm}$ and compared with external AMP and ATP standards to confirm its identity [25].

\section{Live cell intracellular calcium measurements}

The fluorescent calcium indicator, fura-2-acetoxymethyl ester (fura-2-AM; Molecular Probes, Eugene, OR), was used to measure changes in intracellular free calcium $\left(\left[\mathrm{Ca}^{2+}\right]_{\mathrm{i}}\right)$. Cells were incubated at $25^{\circ} \mathrm{C}$ for $60 \mathrm{~min}$ with 1 $\mu \mathrm{M}$ fura-2- $\mathrm{AM}$ in modified Krebs Ringer buffer [(in mM) $120 \mathrm{NaCl}, 5 \mathrm{KCl}, 1.2 \mathrm{CaCl}_{2}, 0.7 \mathrm{MgSO}_{4}, 15 \mathrm{~N}-2$ hydroxyethylpiperazine-N'-2-ethanesulfonic acid (HEPES), and $1.8 \mathrm{~g} / \mathrm{L}$ glucose (pH 7.4)]. After dye loading, cells were washed three times in the same medium and kept in the dark for at least $30 \mathrm{~min}$ before singlecell $\left[\mathrm{Ca}^{2+}\right]_{\mathrm{i}}$ measurements. Changes in $\left[\mathrm{Ca}^{2+}\right]_{\mathrm{i}}$ were determined ratiometrically $(340 \mathrm{~nm} / 380 \mathrm{~nm}$ excitation, $512 \mathrm{~nm}$ emission) in 1-mL aliquots using a spectrofluorometer. Calcium concentrations were calculated using the equation: $\left[\mathrm{Ca}^{2+}\right]_{\mathrm{i}}=\mathrm{K}_{\mathrm{d}}\left(\mathrm{F}_{380 \max } / \mathrm{F}_{380 \min }\right)\left(\mathrm{R}-\mathrm{R}_{\min }\right) /$ $\left(\mathrm{R}_{\max }-\mathrm{R}\right)$ [26]. A dissociation constant $\left(\mathrm{K}_{\mathrm{d}}\right)$ value of $224 \mathrm{nM}$ was assumed for the binding of calcium to fura-2-AM. $R_{\max }$ and $R_{\text {min }}$ were determined in each experimental group by the consecutive addition of 30 $\mu \mathrm{M}$ digitonin $\left(\mathrm{R}_{\max }\right)$ and $50 \mathrm{mM}$ EGTA $\left(\mathrm{R}_{\min }\right) . \mathrm{R}_{\max }$ and $R_{\text {min }}$ are the maximum and minimum $F_{340} / F_{380}$ ratios, respectively. $F_{380 \text { max }} / F_{380 \text { min }}=$ the ratio of fluorescence emission intensity at $380-\mathrm{nm}$ excitation in $\mathrm{Ca}^{2}$ ${ }^{+}$-depleting $\left(\mathrm{F}_{380 \max }\right)$ and $\mathrm{Ca}^{2+}$-saturating $\left(\mathrm{F}_{380 \mathrm{~min}}\right)$ conditions. 


\section{SiRNA transfection}

AMPK siRNA SMARTpoolTM were purchased from Upstate Biotechnology (Chicago, IL). A nonrelated, scrambled siRNA pool (Dharmacon; Lafayette, CO) was used as a negative control. For AMPK knockdown, HepG2 cells were plated in 6-well plates and transfected with $50 \mathrm{nM}$ siRNA (AMPK SMARTpool or nonspecific siRNA pool) using Oligofectamine reagent (Invitrogen; cat. no. 12252-011) following the manufacturer's instruction. Real-time PCR was used to evaluate AMPK knockdown efficiency after cells were incubated in transfection medium for $48 \mathrm{~h}$.

\section{Quantitative Real-Time PCR}

Total RNAs were extracted from HepG2 cells using TRIzol reagent (Invitrogen) according to the manufacturer's protocol. The reverse-transcription reaction was performed using $1 \mu \mathrm{g}$ total RNA that was reverse-transcribed into the first-strand cDNA by Superscript II reverse transcriptase with random primers (Invitrogen). The cDNA levels were quantified by real-time PCR with SYBR Green using a Platinum qRT-PCR kit (Invitrogen) on an ABI 7700 sequence detection system (PerkinElmer Biosystems). Primers are shown as follows: human CPT1 (Accession no. NM_001876), Forward: 5'CGTCTTTTGGGATCCACGATT-3', Reverse: 5'TGTGCTGGATGGTGTC TGTCTC-3'; human FAS (Accession no. NM_004104), Forward: 5'CTGTCTAGGTTTGATG CCTCCT -3', Reverse: 5'GATCCGAGGGCCTCACTAAAC-3'. Reactions were carried out at least in duplicate for each sample on an ABI 7700 sequence detection system (PerkinElmer Biosystems) according to the manufacturer's instructions, and target values were normalized to glyceraldehyde-3phosphate dehydrogenase (GAPDH) as indicated. Exponential amplification efficiency was verified during each PCR run using a standard dilution series made from pooled samples. Results were calculated from the difference in threshold cycle values for CPT-1, FAS and GAPDH.

\section{Quantification malonyl-CoA}

For quantification of malonyl-CoA in cells, radiolabeled palmitate stoichiometrically converted from malonyl$\mathrm{CoA}$ in the presence of $\left[{ }^{14} \mathrm{C}\right]$ acetyl-CoA, and purified fatty acid synthetase (FAS) was measured using a modification of a previously described method [27]. The radiolabeled palmitoyl-CoA was extracted with hexane, and radioactivity was measured using a liquid scintillation counter (Canberra-Packard, Zellik, Belgium).

\section{Measurement of triglyceride content}

To assay triglyceride contents in HepG2 cells, $30 \mu \mathrm{L}$ of triglyceride standard or cleared cell supernatant was added to a 96-well flat bottom polystyrene plate, and $300 \mu \mathrm{L}$ of infinity triglyceride reagent was then added to the microplate. After cooling to room temperature, samples were read at $520 \mathrm{~nm}$ with a Microplate Spectrophotometer (Bio-Tek Instruments Inc., Winooski, VT). Triglyceride levels were normalized to protein concentrations and expressed as $\mu \mathrm{g}$ of lipid/mg of protein.

\section{Cytotoxicity assay}

HepG2 cells $\left(1 \times 10^{4}\right.$ cells $)$ were plated in 96-well plates and cultured for $12 \mathrm{~h}$ in DMEM containing 10\% FBS. The cells were then treated with Cy-3-g or staurosporine at the concentrations of $0.001 \sim 100 \mu \mathrm{M}$ for $16 \mathrm{~h}$. The cell viability was determined by WST assay using Cell Proliferation Reagent WST-1 (Roche). Plates were incubated at $37^{\circ} \mathrm{C}$ to allow for color development and cell viability was evaluated by conversion of a tetrazolium salt into a formazon product measured spectrophotometrically at $490 \mathrm{~nm}$.

\section{Fatty acid $\beta$-oxidation}

HepG2 cells treated with vehicle or Cy-3-g were incubated with $500 \mathrm{mM}$ palmitic acid/BSA in maintenance medium for $24 \mathrm{~h}$ followed by incubation with $125 \mathrm{mM}$ $\left[{ }^{3} \mathrm{H}\right]$ palmitic acid/BSA and $1 \mathrm{mM}$ carnitine in PBS for 2 h. ${ }^{3} \mathrm{H}_{2} \mathrm{O}$ was measured as described previously [4].

\section{Fatty acid synthesis assay}

HepG2 cells $\left(5 \times 10^{4}\right.$ per well $)$ were plated on Costar black-walled 96-well assay plate and pretreated as previously described [28]. After 16-hour incubation, the pretreated medium was removed and replaced with medium containing $\left[{ }^{14} \mathrm{C}\right]$ acetate $(2 \mu \mathrm{Ci} / \mathrm{mL}$, Perkin Elmer) and AICAR or Cy-3-g at the indicated concentrations. Cells were incubated $4 \mathrm{~h}$ at $37^{\circ} \mathrm{C}$ then the plates were rinsed with $\mathrm{PBS}$. The final wash was replaced with Microscint20 (Perkin Elmer) and radioactivity incorporated into fatty acid monitored on MicroBeta Liquid Scintillation and Luminescence Counters.

\section{Western blot}

Cellular protein extracts were prepared with lysis buffer (50 mM Tris- $\mathrm{HCl}, \mathrm{pH} 8.0,150 \mathrm{mM} \mathrm{NaCl}, 0.5 \%$ NP40) containing complete protease and phosphatase inhibitors (Roche Applied Science, Penzberg, Germany), and then immunoblotted with antibodies against p-AMPK, AMPK, and p-ACC, ACC for $2 \mathrm{~h}$. Specific antibody binding was detected by horseradish peroxidase-conjugated secondary antibodies and visualized using enhanced chemiluminescence detection reagent (Santa Cruz). The band densities were quantified using an image analyzer Quantity One System (Bio-Rad, Richmond, CA). All protein quantifications were adjusted for 
the corresponding $\beta$-actin level, which was not consistently changed by the different treatment conditions.

\section{Statistical analysis}

Statistical analyses were performed using the SPSS 14.0 package (SPSS Inc., Chicago, IL). All results are expressed as means \pm SE. and analyzed by the Student's $t$ test or analysis of variance (ANOVA) to determine $P$ values; $P<0.05$ was considered statistically significant.

\section{Abbreviations \\ ACC: acetyl-CoA carboxylase; AMPK: AMP-activated protein kinase; CaMKK calmodulin-dependent protein kinase kinase; CPT1: carnitine palmitoyl-CoA transferase 1; Cy-3-g: cyanidin-3-O-B-glucoside; FAS: fatty acid synthase; FBPase: fructose-1,6 diphosphatase; GPPase: glycogen phosphorylase; MCD: malonyl CoA decarboxylase; NAFLD: nonalcoholic fatty liver disease; TG: triglyceride}

\section{Acknowledgements}

This work was supported by grants from the National Natural Science Foundation (81172655), a Foundation for Qualified Personnel in Colleges and Universities in Guangdong Province (2011-128), the Research Fund Program of Guangdong Provincial Key Laboratory of Food, Nutrition and Health (2011K-003).

\section{Author details}

'Department of Food Science, Yingdong College of Bioengineering, Shaoguan University, Shaoguan, Guangdong Province, China. ${ }^{2}$ Guangdong Provincial Key Laboratory of Food, Nutrition and Health; Department of Nutrition, School of Public Health, Sun Yat-sen University (Northern Campus), Guangzhou, Guangdong Province, China.

\section{Authors' contributions}

HG and MX conceived the idea and designed the study. DW and GL carried out the lipid metabolism assay experiments. RZ performed the statistical analysis and interpretation of the data. YW participated in the experiments related to AMPK enzyme activity assay. HG drafted the manuscript and MX provided critical corrections to the manuscript. All authors read and approved the final manuscript.

\section{Competing interests}

The authors declare that they have no competing interests.

Received: 5 December 2011 Accepted: 13 January 2012

Published: 13 January 2012

\section{References}

1. Cohen JC, Horton JD, Hobbs HH: Human fatty liver disease: old questions and new insights. Science 2011, 332:1519-1523.

2. Lam B, Younossi ZM: Treatment options for nonalcoholic fatty liver disease. Therap Adv Gastroenterol 2010, 3:121-137.

3. Long YC, Zierath JR: AMP-activated protein kinase signaling in metabolic regulation. J Clin Invest 2006, 116:1776-1783.

4. Lopez M, Lelliott CJ, Tovar S, Kimber W, Gallego R, Virtue S, Blount M, Vazquez MJ, Finer N, Powles TJ, O'Rahilly S, Saha AK, Dieguez C, VidalPuig AJ: Tamoxifen-induced anorexia is associated with fatty acid synthase inhibition in the ventromedial nucleus of the hypothalamus and accumulation of malonyl-CoA. Diabetes 2006, 55:1327-1336.

5. Assifi MM, Suchankova G, Constant S, Prentki M, Saha AK, Ruderman NB: AMP-activated protein kinase and coordination of hepatic fatty acid metabolism of starved/carbohydrate-refed rats. Am J Physiol Endocrinol Metab 2005, 289:E794-800

6. He J, Giusti MM: Anthocyanins: natural colorants with health-promoting properties. Annu Rev Food Sci Technol 2010, 1:163-187.

7. Zern TL, Wood RJ, Greene C, West KL, Liu Y, Aggarwal D, Shachter NS, Fernandez ML: Grape polyphenols exert a cardioprotective effect in pre- and postmenopausal women by lowering plasma lipids and reducing oxidative stress. The Journal of nutrition 2005, 135:1911-1917.

8. Qin Y, Xia M, Ma J, Hao Y, Liu J, Mou H, Cao L, Ling W: Anthocyanin supplementation improves serum LDL- and HDL-cholesterol concentrations associated with the inhibition of cholesteryl ester transfer protein in dyslipidemic subjects. The American journal of clinical nutrition 2009.

9. Guo H, Ling W, Wang Q, Liu C, Hu Y, Xia M, Feng X, Xia X: Effect of anthocyanin-rich extract from black rice (Oryza sativa L. indica) on hyperlipidemia and insulin resistance in fructose-fed rats. Plant Foods Hum Nutr 2007, 62:1-6.

10. Peng CH, Liu LK, Chuang CM, Chyau CC, Huang CN, Wang CJ: Mulberry water extracts possess an anti-obesity effect and ability to inhibit hepatic lipogenesis and promote lipolysis. J Agric Food Chem 2011, 59:2663-2671.

11. Prior RL, S EW, T RR, Khanal RC, Wu X, Howard LR: Purified blueberry anthocyanins and blueberry juice alter development of obesity in mice fed an obesogenic high-fat diet. J Agric Food Chem 2010, 58:3970-3976.

12. Guo H, Xia M, Zou T, Ling W, Zhong R, Zhang W: Cyanidin 3-glucoside attenuates obesity-associated insulin resistance and hepatic steatosis in high-fat diet-fed and $\mathrm{db} / \mathrm{db}$ mice via the transcription factor FoxO1. Nutr Biochem 2011, DOl10.1016/j.jnutbio.2010.12.013.

13. Cool B, Zinker B, Chiou W, Kifle L, Cao N, Perham M, Dickinson R, Adler A Gagne G, lyengar R, Zhao G, Marsh K, Kym P, Jung P, Camp HS, Frevert E: Identification and characterization of a small molecule AMPK activator that treats key components of type 2 diabetes and the metabolic syndrome. Cell Metab 2006, 3:403-416.

14. Hurley RL, Anderson KA, Franzone JM, Kemp BE, Means AR, Witters LA: The $\mathrm{Ca} 2+/$ calmodulin-dependent protein kinase kinases are AMP-activated protein kinase kinases. J Biol Chem 2005, 280:29060-29066.

15. Obici S, Feng Z, Arduini A, Conti R, Rossetti L: Inhibition of hypothalamic carnitine palmitoyltransferase-1 decreases food intake and glucose production. Nat Med 2003, 9:756-761.

16. Ruderman NB, Saha AK, Kraegen EW: Minireview: malonyl CoA, AMPactivated protein kinase, and adiposity. Endocrinology 2003, 144:5166-5171.

17. Gaidhu MP, Fediuc S, Ceddia RB: 5-Aminoimidazole-4-carboxamide-1beta-D-ribofuranoside-induced AMP-activated protein kinase phosphorylation inhibits basal and insulin-stimulated glucose uptake, lipid synthesis, and fatty acid oxidation in isolated rat adipocytes. J Biol Chem 2006, 281:25956-25964.

18. Tsuda T, Ueno Y, Aoki H, Koda T, Horio F, Takahashi N, Kawada T, Osawa T: Anthocyanin enhances adipocytokine secretion and adipocyte-specific gene expression in isolated rat adipocytes. Biochem Biophys Res Commun 2004, 316:149-157.

19. Tsuda T: Regulation of adipocyte function by anthocyanins; possibility of preventing the metabolic syndrome. J Agric Food Chem 2008, 56:642-646.

20. Takikawa M, Inoue S, Horio F, Tsuda T: Dietary anthocyanin-rich bilberry extract ameliorates hyperglycemia and insulin sensitivity via activation of AMP-activated protein kinase in diabetic mice. J Nutr 2010, 140:527-533

21. Woods A, Johnstone SR, Dickerson K, Leiper FC, Fryer LG, Neumann D, Schlattner U, Wallimann T, Carlson M, Carling D: LKB1 is the upstream kinase in the AMP-activated protein kinase cascade. Curr Biol 2003, 13:2004-2008.

22. Kahn BB, Alquier T, Carling D, Hardie DG: AMP-activated protein kinase: ancient energy gauge provides clues to modern understanding of metabolism. Cell Metab 2005, 1:15-25.

23. Kaiser A, Nishi K, Gorin FA, Walsh DA, Bradbury EM, Schnier JB: The cyclindependent kinase (CDK) inhibitor flavopiridol inhibits glycogen phosphorylase. Arch Biochem Biophys 2001, 386:179-187.

24. Dougherty MJ, Boyd JM, Downs DM: Inhibition of fructose-1,6bisphosphatase by aminoimidazole carboxamide ribotide prevents growth of Salmonella enterica purH mutants on glycerol. J Biol Chem 2006, 281:33892-33899.

25. Luo B, Parker GJ, Cooksey RC, Soesanto Y, Evans M, Jones D, McClain DA: Chronic hexosamine flux stimulates fatty acid oxidation by activating AMP-activated protein kinase in adipocytes. J Biol Chem 2007, 282:7172-7180 
26. Grynkiewicz G, Poenie M, Tsien RY: A new generation of $\mathrm{Ca}$ 2+ indicators with greatly improved fluorescence properties. J Biol Chem 1985, 260:3440-3450.

27. Harwood HJ Jr, Petras SF, Shelly LD, Zaccaro LM, Perry DA, Makowski MR, Hargrove DM, Martin KA, Tracey WR, Chapman JG, Magee WP, Dalvie DK, Soliman VF, Martin WH, Mularski CJ, Eisenbeis SA: Isozyme-nonselective Nsubstituted bipiperidylcarboxamide acetyl-CoA carboxylase inhibitors reduce tissue malonyl-CoA concentrations, inhibit fatty acid synthesis, and increase fatty acid oxidation in cultured cells and in experimental animals. J Biol Chem 2003, 278:37099-37111.

28. Lian Z, Li Y, Gao J, Qu K, Li J, Hao L, Wu S, Zhu H: A novel AMPK activator WS070117, improves lipid metabolism discords in hamsters and HepG2 cells. Lipids Health Dis 2011, 10:67.

doi:10.1186/1476-511X-11-10

Cite this article as: Guo et al: Cyanidin-3-O- $\beta$-glucoside regulates fatty acid metabolism via an AMP-activated protein kinase-dependent signaling pathway in human HepG2 cells. Lipids in Health and Disease 2012 11:10

\section{Submit your next manuscript to BioMed Central} and take full advantage of:

- Convenient online submission

- Thorough peer review

- No space constraints or color figure charges

- Immediate publication on acceptance

- Inclusion in PubMed, CAS, Scopus and Google Scholar

- Research which is freely available for redistribution

Submit your manuscript at www.biomedcentral.com/submit 